\title{
Towards fault detection and self-healing of chemical processes over wireless
}

\section{sensor networks}

\author{
Bogdan Dorneanu, $u^{\mathrm{a}}$ Hang Ruan ${ }^{\mathrm{b}}$, Abdelrahim Mohamed ${ }^{\mathrm{b}}$, Mohamed Heshmat ${ }^{\mathrm{c}, \mathrm{d}}$, Yang $\mathrm{Gao}^{\mathrm{c}}$, \\ Pei Xiao ${ }^{\mathrm{b}}$, Harvey Arellano-Garcia ${ }^{\mathrm{a}}$
}

\author{
${ }^{a}$ Department of Chemical and Process Engineering, University of Surrey, Guildford, GU2 7XH, UK \\ ${ }^{b}$ Institute for Communication systems (ICS), Home of 5GIC, University of Surrey, Guildford, GU2 7XH, UK \\ ${ }^{c}$ Surrey Space Centre, University of Surrey, Guildford, GU2 7XH, UK \\ ${ }^{d}$ Department of Mathematics, Faculty of Science, Sohag University, Sohag, Egypt
}

ABSTRACT: This contribution introduces a framework for the fault detection and healing of chemical processes over wireless sensor networks. The approach considers the development of a hybrid system which consists of a fault detection method based on machine learning, a wireless communication model and an ontologybased multi-agent system with a cooperative control for the process monitoring.

\section{INTRODUCTION}

Modern engineering systems and manufacturing processes are becoming increasingly complex, and operating in highly dynamic environments. In the age of high competition and stringent environmental and safety regulations, the role of maintenance as an effective tool to increase profit margin, improve plant reliability and reduce safety and environmental hazards has become increasingly important (Nguyen \& Bagajewicz, 2010). Fault detection and diagnosis are important issues in chemical engineering applications. The early detection of process faults can help avoid or reduce productivity loss and its associated costs. The petrochemical industry, for example, loses an estimated of 20 billion dollars every year and rated abnormal event management (AEM) as their number one problem that needs to be solved (Venkatasubramanian et al. 2003). Research conducted by Oneserve in partnership with British manufacturers found that $3 \%$ of all working days are lost annually in manufacturing due to faulty machinery, amounting to a cost of $£ 180$ billion a year.

In industrial processes faults happen due to design error, implementation errors, human operator errors, wear, aging, or environmental aggressions. A fault can be defined as a deviation of at least one characteristic property or parameter of the system from the standard condition. Fault diagnosis is a subfield of control engineering which includes fault detection, fault isolation, and fault identification. Fault detection techniques work on capturing the fault and estimating the time of fault occurrence. Then it comes to the fault isolation to determine the location of the fault in the system and the fault identification to estimate the size and the type of fault (Severson et al. 2015). Fault detection and isolation (FDI) methods can be classified into (Zhong et al. 2018): a) modelbased methods, which provide a description of the dynamic behaviour and a physical understanding of the system, but are difficult to account for modelling errors and uncertainties; b) knowledge-based methods, which combine rules derived from first principles, rules with no underlying first principles from physics and simple limit checks; and c) data-driven/signalbased methods, often used in monitoring of largescale industrial applications as they do not require a lot of computation and are compatible with real-time constraints of dynamic complex systems, but require pre-processing steps for extracting information on the system. In the following, a novel framework for fault detection in chemical processes will be presented, based on a combination of ontology-based multiagent systems, cooperative MPC, communications over wireless sensor networks and fault detection using machine learning algorithms.

The reminder of this paper is structured as follows: Section 2 introduces the communication network and data transfer scheme. Section 3 provides an overview of multi-agent systems (MAS) and ontology for fault detection, followed by machine learning techniques for fault detection in Section 4. Section 5 introduces the overall system architecture combining the latest wireless communication techniques with fault detection. Finally, conclusions are drawn in Section 6.

\section{COMMUNICATION NETWORKS AND DATA TRANSFER}

Wireless sensors are considered as one of the key enables in industry and process automation, more specifically in intelligent chemical/process plants. Unlike conventional wired sensors, wireless sensors can be deployed in hazardous areas of the plant in a plugand-play mode and they reduce deployment and maintenance overhead. It is envisioned that many sensors will be deployed in large-scale plants to provide reliable, timely and up-to-date measurements, thus enabling fast and accurate decisions by the control system. Some of these sensors may provide periodic measurements with different inter-measurement times, i.e. traffic inter-arrival times, while others will be configured to operate on an event-basis. For instance, a temperature sensor can be configured to 
transmit measurement when a certain condition is met and suspends transmission otherwise. In such scenarios, reliable network access, massive connectivity support and timely scheduling will become critical considerations.

The legacy wireless systems have been designed primarily for human initiated mobile broadband communications. They are highly suboptimal for latencycritical, narrow band, short-bust, sporadic traffic (e.g. measurement data, such as temperature, pressure, humidity, etc.) generated by sensors. Consequently, a new design paradigm is needed to support large numbers of heterogeneous sensing devices with diverse requirements and unique traffic characteristics. Compared to the sensors in traditional Internet-of-Things (IoT) networks, those deployed in extreme environments need to operate in harsh (sometimes hazardous) conditions, thus are prone to wear and tear, and cannot be easily replaced, posing major challenges in designing resilient wireless networks for reliable communications.

Recently, the first release of the fifth-generation (5G) cellular system has been standardised, and key use cases for narrow-band communications have been identified for ultra-reliable and low latency communications (URLLC) and massive machine-type communications (mMTC). The former is characterised by extremely low end-to-end transmission latencies (1 ms user plane latency and $20 \mathrm{~ms}$ control plane lantecy) and high reliability figures (e.g. $99.999 \%$ success rate within $1 \mathrm{~ms}$ with $0 \mathrm{~ms}$ mobility interruption time), while the latter supports extremely high connection densities up to $1,000,000$ devices per $\mathrm{km}^{2}$ with typically low data rates (ITU, 2017; Nokia, 2018).

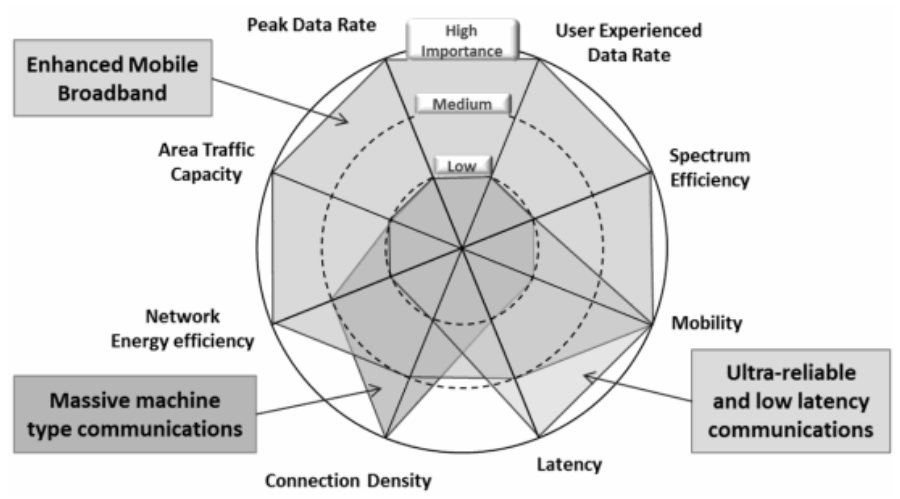

Fig.1: $5 G$ use cases (3GPP, 2015)

Fig. 1 maps the main parameters of each $5 \mathrm{G}$ use case. It can be noticed that low latency and high connection density are the main requirements for the scenarios under consideration for plant maintenance. For instance, the fault detection and prediction algorithm will require fast transmission of measurement data to provide timely decisions. In addition, it is envisioned that large number of sensors will be deployed within a plant, and the same network can support multiple plants/factories. In other words, both the mMTC and the URLLC system characteristics should be taken into account for the scenarios under investigation.

\section{MULTI-AGENT SYSTEMS AND ONTOLOGY FOR FAULT DETECTION}

Ontologies and multi-agent systems (MAS) can be used in the development of knowledge-based FDI techniques. An ontology is a formal representation of a set of concepts within a domain and the relationships between those concepts. One of the advantages of ontologies is that they can be processed by logic reasoners or inference engines so that hidden relationships between elements of a system can be discovered. The ontology serves as a library of knowledge components to efficiently build intelligent systems and as a shared vocabulary for communication between interacting human and/or software agents (Batres, 2017).

Furthermore, an agent can be defined as an entity which is placed in an environment and senses different parameters that are used to make a decision based on the goal of the entity. The MAS is a computerised system composed of multiple interacting agents exploited to solve a problem. The salient features of a MAS, which include efficiency, low cost, flexibility, and reliability, make it an effective solution to solve complex tasks (Dorri et al. 2018).

Ontology and MAS have been often used in development of FDI methodologies. One of these approaches includes information contained in the operating, safety and control procedures for diagnosis of complex process plants (Hangos et al. 2008). A MAS technology-based chemical plant supervisory system has been developed that connects the chemical equipment and is able to monitor the entire enterprise (Wang \& Zhang, 2008). The proposed system can also be integrated with the current systems through and interface.

Other approaches of ontology and MAS-based process supervision have been developed using a conceptualisation of equipment, control systems and hazards, or to demonstrate how description logic (DL) reasoning could be used to detect and diagnose faults, without the help of external agents (Musulin et al, 2013).

For the management of abnormal situations, an agentbased approach called ENCORE, using OntoSafe, explicitly captures the hierarchy of the offshore platform, comprising the entire process at the highest level and the individual instruments and equipment at the lowest (Natarajan \& Srinivasan, 2014). Each FDI method in ENCORE is modelled as an agent specialised in monitoring different aspects at varying levels and scope.

A robust fault detection techniques based on consensus-based multi-agent approach for sensors networks 
makes use of the information interaction and coordination among the neighbouring networks for the fault detection (Jiang et al. 2014).

More recently, a framework for automatic generation of a flexible and modular system has been proposed for fault detection and diagnosis (Steinegger et al. 2017). This methods gathers the information from various engineering artefacts using ontology and generates fault detection and diagnosis functions based on structural and procedural generation rules.

\section{MACHINE LEARNING FOR FAULT DETECTION}

There are two types of machine learning (ML) methods towards fault detection. a) Supervised learning (SL) approaches (Wang et al. 2015), where the fault detection ML model needs to be trained with some expert knowledge. This means that there should be some previous information on how faulty the data is. Most real scenarios, this prerequisites are not available, as the faults are not known until they are detected, recorded and analysed for the first time. b) Unsupervised learning (USL) approaches (Costa et al. 2014), which do not depend on expert knowledge of faults. USL includes options such as clustering (Jyoti \& Singh, 2011) or self-organising map (SOM) methods (Chen \& Yan, 2012). However, very limited USL methods deliver effective performance due to the high complexity cost, especially for those hierarchical approaches, which force the algorithms to run over and over again on pairwise samples of large datasets. In most scenarios, due to limitation of hardware, the hierarchical approaches are infeasible as the hardware cost would be much higher and the performance are still unpredictable.

\section{FAULT DETECTION VIA WIRELESS SENSOR NETWORKS}

As no one fault detector can work well for complex processes (Venkatasubramanian, V. et al. 2003; Miljkoviç, 2011), a hybrid algorithm is included in the framework, illustrated in Fig.2, for robust fault detection capability.

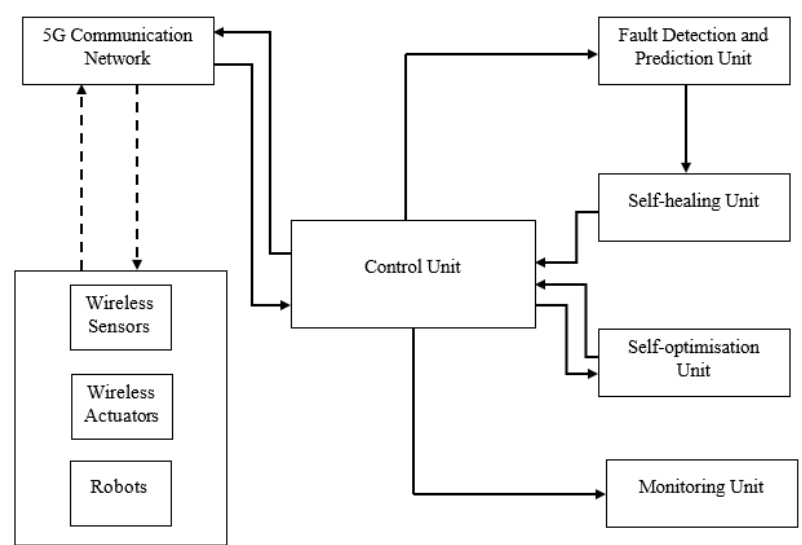

Fig. 2: High level overview of the overall system model
The framework consists of a network of four main components. The first one is a wireless sensors that facilitate data management for better data-based fault detection. The wireless sensors network (WSN) transmits over a $5 \mathrm{G}$ communication network. The second component is an efficient fault detection algorithm that could analyse the data and classify it in faulty or normal. The third component is a knowledge-based and a model-based fault detection monitoring system, based on ontology and MAS. Finally, the fourth component is a cooperative MPC system that takes the required measures to ensure stable process operation.

Using information on the process' structure and behaviour, equipment information, and expert knowledge encoded into the ontology, the system is able to detect any faults. The integration with the monitoring system will facilitate the detection and optimise the controller's actions.

A low data rate is considered for all sensors. In addition, most sensors are assumed to be static, i.e. deployed in fixed locations. Since humanoid robots and autonomous robots can be considered as an element of the control and measurement system, it is assumed that a small number of sensors are moving with a low speed (e.g. $\leq 3 \mathrm{~km} / \mathrm{h}$ ).

A centralised control mechanism is considered where the sensors are connected to a fusion node via wireless links.

The latter can also be used to send commands to actuators within the plant. Consequently, the measurements are transmitted in the uplink to the control unit with the commands being transmitted in the downlink. The network consists of a heterogeneous set of periodic and event-triggered sensors with mixed requirements, characteristics and traffic models.

Considering heterogeneity of the plant and the associated sensors, a statistical model rather than a deterministic model is chosen for the sensor transmission events. The number of incoming packets (or events when each event generates a single packet) per unit of time follows the Poisson distribution while the packet interval is modelled as an exponential distribution.

This results in probability-based transmissions that can be controlled by the arrival rate and the inter-arrival time. Fig. 3 shows an example of the probabilitybased transmission.

Given this traffic model coupled with the network model illustrated in Fig.2, the fault detection framework will propose a low latency network access scheme for wireless sensors networks in intelligent chemical/process plants. The proposed scheme considers the $5 \mathrm{G}$ frame structure and allows massive numbers of sensors to access the network simultaneously without collisions to request resources for measurement transmission.

A low overhead and low latency approach is being developed, and simulation along with theoretical evaluations are used to show gains of the proposed over conventional access schemes. 
Event arrival rate $=2$ ( 1 event per $0.5 \mathrm{~min})$

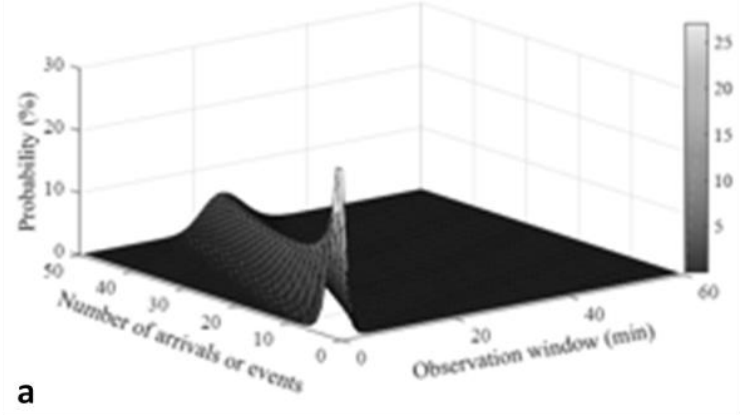

Event arrival rate $=0.2(1$ event per 5 min $)$

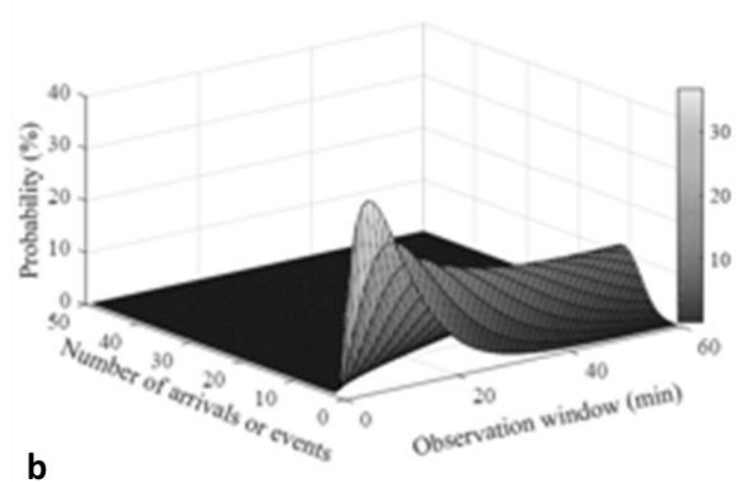

Event arrival rate $=0.033(1$ event per $30 \mathrm{~min})$

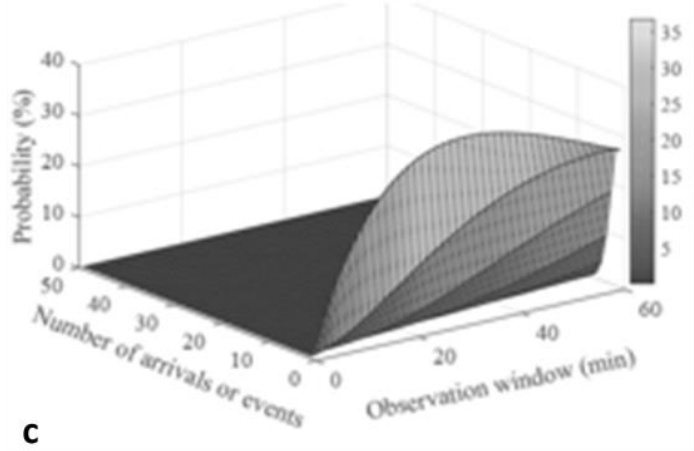

Fig.3: Transmission probability for:

a) high periodicity sensor/probability event; $b$ ) medium periodicity sensor/probability event; c) low periodicity sensor/probability event.

Once the measurement data is received at the network side, it will be routed to the control unit for further processing. This data can be used for fault detection and prediction, as well as to optimise the plant operating parameters.

Decisions of the control unit will be routed to the $5 \mathrm{G}$ base station (known as g-NodeB), from where the commands will be sent to the wireless actuators.

The control unit is developed based on cooperative control algorithm, based on distributed MPC approaches (Rocha \& Oliveira, 2016).

Under the cooperative framework, the distributed controllers share information about their state with other controllers, as shown in Fig.4. Thus, the interactions between the processing units will not be lost, as the local controller will optimise a local objective function, with input from other sections of the process.

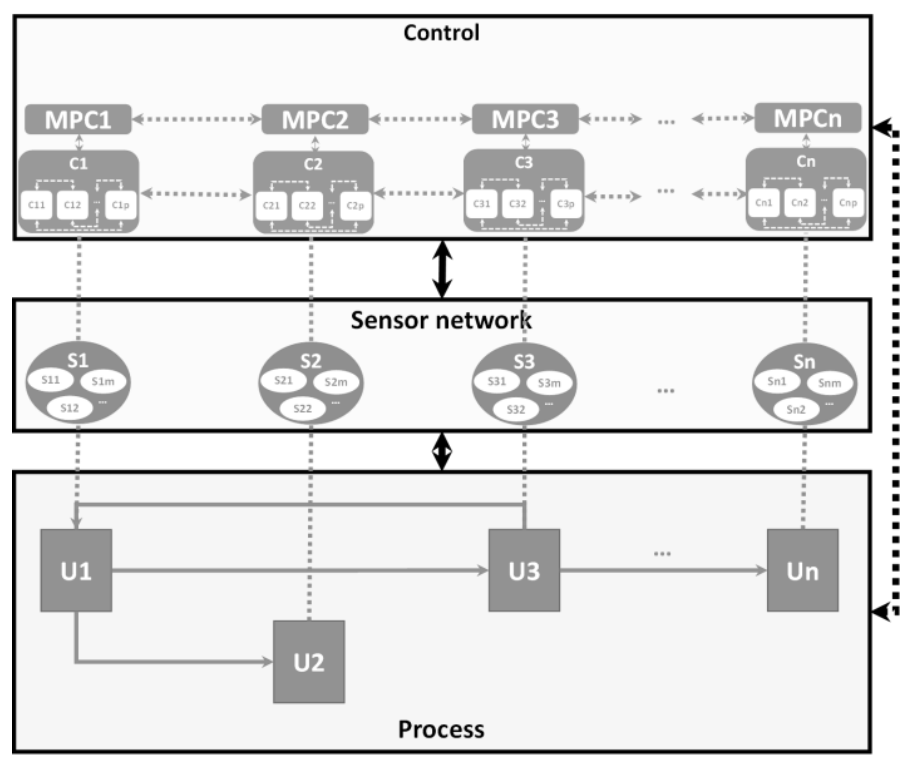

Fig. 4: Cooperative control framework

For the fault detection, a two-stage method based on a hybrid learning approach is applied, which utilises both SL and USL. In the first stage, an unsupervised learning algorithm (a K-aware K-mean - KKM - clustering) is applied to separate the whole dataset into clusters based on their similarities and select the optimal $\mathrm{K}$ value (within a given range) for the best clustering performance. The cluster with the smallest amount of data which is also much smaller than the penultimate cluster, is perceived as the faulty data cluster. The proposed KKM-clustering method is summarised in Fig.5.

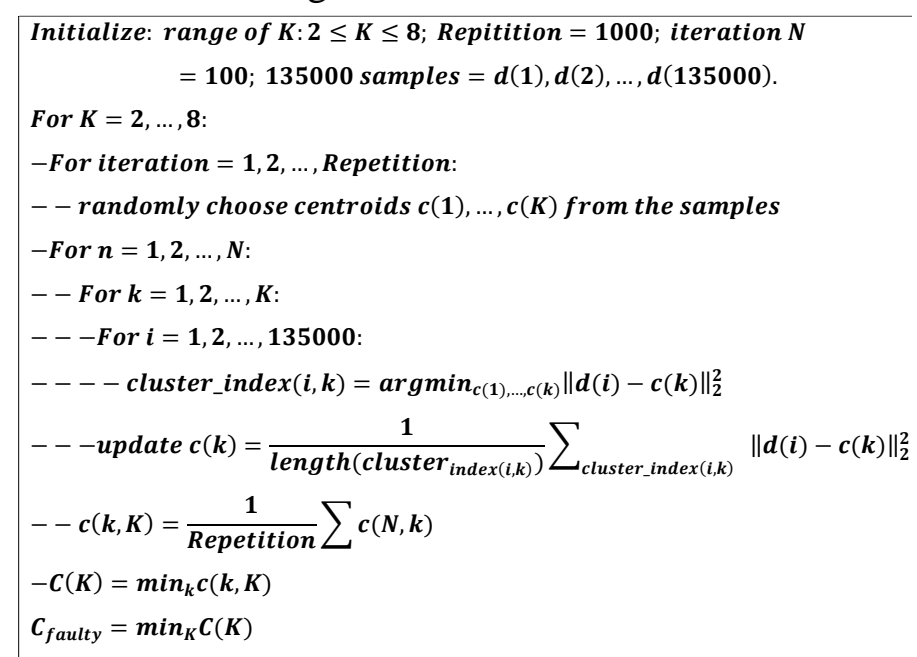

\section{Fig.5: KKM clustering algorithm}

The value of $\mathrm{K}$ varies only between 2 and 8 . For each value of $\mathrm{K}$, the algorithm is repeated 1,000 times, for reproducible results. In each repetition, 100 iterations are conducted to iteratively compute and update the centroids and cluster indices of the data clusters. Then, at each $\mathrm{K}$-mean algorithm for each $\mathrm{K}$ value, the 
smallest data cluster is selected. Finally, the selected cluster with the smallest size is perceived as the faulty data cluster, while the corresponding value of $\mathrm{K}$ is selected as the best $\mathrm{K}$. Please not that $\|\cdot\|_{2}$ denotes the Euclidean distance, cluster_index $(i, k)$ the distance between sample $i$ and cluster $k$, used to index both data points and the clusters they are located in, $c(k)$ the index of cluster $k, c(N, k)$ the cluster $k$ after $N$ iterations, $c(k, K)$ the cluster $k$ with a certain value of $K, C(K)$ the smallest cluster with a certain value of $K$, and $C_{\text {faulty }}$ is the perceived faulty data cluster.

In the second stage, a single SL method is used for training on the pre-processed data obtained from stage 1 , and then used to classify faults from the unknown dataset after training. Various classical SL algorithms will be applied, to compare their accuracy. Preliminary results were obtained for a set of data collected from a pilot plant that produces sodium ion solution as sodium chloride for sale to fine chemical, pharmaceutical, and food industry, which is illustrated as the Process in Fig.4. The data was collected from March $21^{\text {st }}-24^{\text {th }}, 2017$, which aggregated $30 \mathrm{MB}$ in total. The data was collected by 43 fixed sensors set up on the units of the pilot plant with a frequency of $1 \mathrm{~Hz}$ (i.e. one sample per second). A total collection time of $8 \mathrm{~h}$ was considered for each day, which delivered $135 \mathrm{k}$ data samples. Every sample measured 43 variables, consistent with the number of installed sensors. Data points include both floating point and Boolean data. For stage 2, the whole dataset with labels is split into training and testing subsets of $100 \mathrm{k}$ and $35 \mathrm{k}$, respectively for SL so that a specific fault detection ML model can be established.

For the pre-processing of the raw data, measurements are standardised using the standard scalarization method to remove the means. In order to have an intuitive visualisation of the data, the principal component analysis (PCA) is used to project the original unvisualisable data from higher-order feature space onto a 2D plane based on its first two principal eigen components, illustrated in Fig.6.

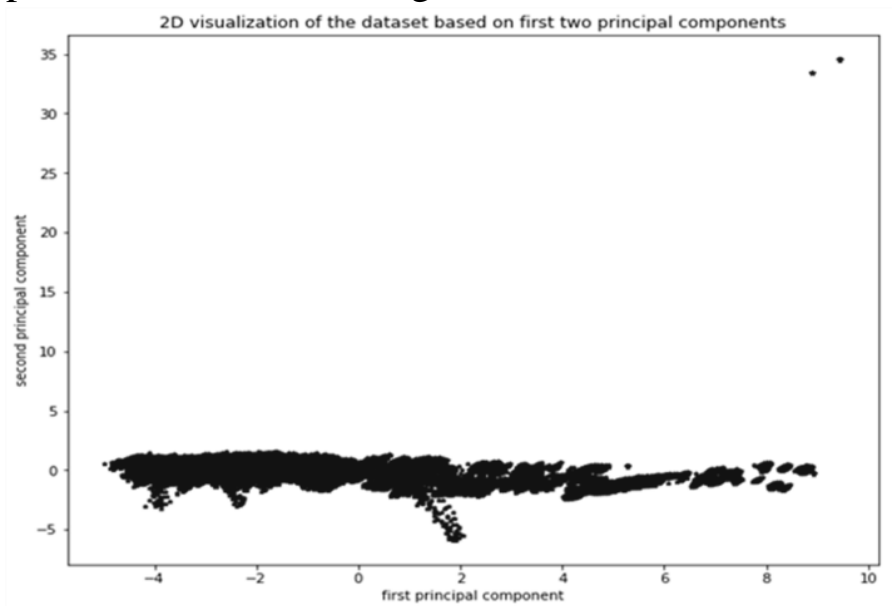

Fig.6: Data visualisation after applying PCA with 2 principal components
Most of the data is distributed with the second principal component value less than 2, except for some outliers on the top right corner which have values larger than 30. The proposed KKM algorithm is then used to detect the outliers and categorise them as faults. A mini-batch data packaging method is used to reduce the computational complexity in a moderate level, where the samples are packaged into small batches. Thus, the algorithm has to be run on different subsets of the original data instead of all of them. The minibatch size is set to 1,000 , which only takes 1,000 samples per iteration instead of the 135k. Fig. 2 shows the results after applying the KKM algorithm for the data in stage 1. As can be seen, the outliers (in crosses) are correctly detected. The result also indicates the optimal $\mathrm{K}$ value is 4, where the outlier data is represented by the smallest cluster.

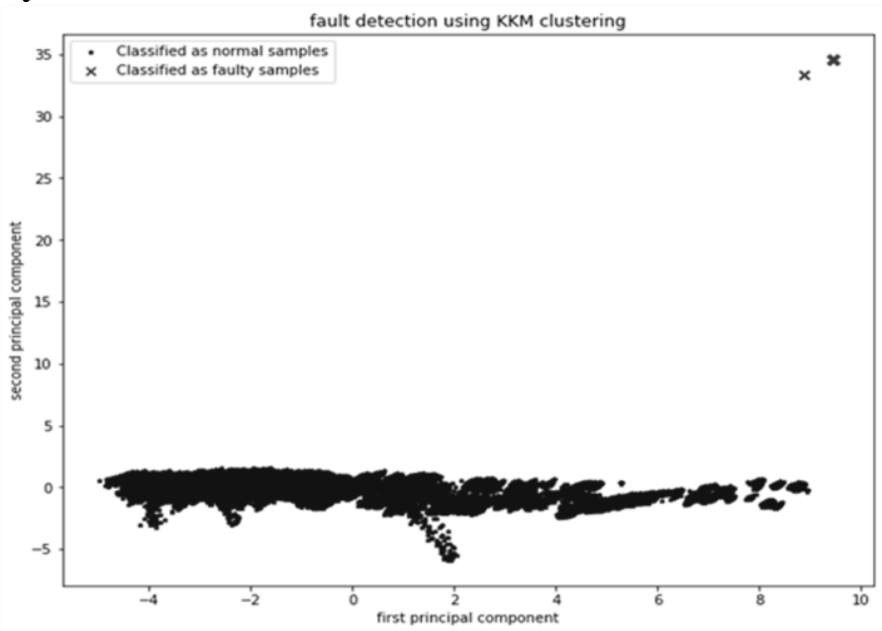

Fig.7: Data visualisation after applying the KKM algorithm

In the $2^{\text {nd }}$ stage, several SL classification algorithms are compared regarding the detection accuracy, based on the expert knowledge obtained from stage 1 . The results are summarised in Table 1. For the multilayer perceptron (MLP) algorithm, only one hidden layer is tested, which proves adequate for the task, while the support vector machines (SVM) only linear kernels are considered for low complexity purposes.

Table 1: Detection accuracy for various classifiers

\begin{tabular}{|c|r|}
\hline Classifier & $\begin{array}{r}\text { Detection } \\
\text { accuracy }\end{array}$ \\
\hline Logistic regression & $97.5 \%$ \\
\hline KNN & $100.0 \%$ \\
\hline DT & $99.4 \%$ \\
\hline Linear discriminant analysis & $95.4 \%$ \\
\hline MLP with one hidden layer (-10-) & $99.0 \%$ \\
\hline Linear SVM & $99.5 \%$ \\
\hline
\end{tabular}

The hyper parameters are properly tuned to produce the best accuracy performance. As shown in Table 1, all algorithms are able to achieve 95\% accuracy, 
where K-nearest neighbours $(\mathrm{KNN})$, decision tree (DT), MLP, and SVM give the best performance, with accuracy $>99 \%$.

\section{CONCLUSIONS}

A framework for the detection of faults and healing in chemical plants is proposed. The framework is developed on an ontology-based MAS that considers models for the physical process, the cooperative MPC system used for controlling the plant, a model for the wireless communication networks between the WSN implemented inside the plant, and a two-stage fault detection approach based on USL and SL. Preliminary results have been obtained for the fault detection within a mini plant operated at the University of Surrey. For the future work, the operation of the plant will be analysed over a longer period of time, under controlled disturbances to assess the healing capabilities of the novel approach. Fault prediction algorithms will be implemented as well within the framework.

\section{ACKNOWLEDGEMENTS}

This project is carried out within the framework of the EP/R001588/1 Stepping towards the industrial $6^{\text {th }}$ Sense project. The financial support of the EPSRC is gratefully acknowledged.

\section{REFERENCES}

3GPP, 2015. Industry vision and schedule for the new radio part of the next generation radio technology, 3GPP RAN workshop on 5G, Phoenix, USA

Batres, R. 2017. Ontologies in process systems engineering, Chemie Ingenieur Technik 89 (11), 14211431

Chen, X. \& Yan, X. 2012. Using improved self-organising map for fault diagnosis in chemical industry process, Chemical Engineering Research and Design 90 (12), 2262-2277

Costa, B.S.J. et al. 2014. A new unsupervised approach to fault detection and identification, Proceedings of the 2014 International Joint Conference on Neural Networks, 1557-1564

Dorri et al. 2018. Multi-agent systems: A survey, IEEE Access 6, 28573-28593

Hangos, K.M. et al. 2008. A procedure ontology for advanced diagnosis of process systems, KnowledgeBased Intelligent Information and Engineering Systems, 501-508

ITU - International Telecommunication Union, 2017, Report ITU-R M.2410.0: Minimum requirements related to technical performance for IMT-2020 radio interface(s)
Jiang, Y. et al. 2014. A consensus-based multi-agent approach for estimation in robust fault detection, ISA Transactions 53 (5), 1562-1568

Jyoti, K. \& Singh, S. 2011. Data clustering approach to industrial process monitoring, fault detection and isolation, International Journal of Computer Applications 17 (2), 41-45

Miljkoviç, D. 2011. Fault detection methods: A literature survey, Proceedings of the $34^{\text {th }}$ International MIPRO Convention, 110-115

Musulin, E. et al. 2013. A knowledge-driven approach for process supervision in chemical plants, Computers \& Chemical Engineering 59, 164-177

Natarajan, S. \& Srinivasan, R. 2014. Implementation of multi agents based system for process supervision in large-scale chemical plants, Computers \& Chemical Engineering 60, 182-196

Nguyen, D. \& Bagajewicz, M. 2010. Optimisation of preventive maintenance in chemical process plants, Industrial \& Engineering Chemistry Research 49 (9), 4329-4339

Nokia, 2018, Self-Evaluation: URLLC and mMTC evaluation results, 3GPP RAN Workshop on 3GPP submission towards IMT-2020, Brussels, Belgium

Rocha, R.R. \& Oliveira-Lopes, L.C. 2016. A cooperative distributed model predictive control for nonlinear systems with automatic partitioning, Computer Aided Chemical Engineering 38, 2205-2210

Severson et al. 2015. Perspective on process monitoring of industrial systems, IFAC-PapersOnLine 48 (21), 931-939

Steinegger, M. et al. 2017. A framework for automatic knowledge-based fault detection in industrial conveyor systems, Proceedings of the $22^{\text {nd }}$ IEEE International Conference on Emerging Technologies and Factory Automation, 1-6

Venkatasubramanian, V. et al. 2003. A review of process fault detection and diagnosis: Part I: Quantitative model-based methods, Computers \& Chemical Engineering 27 (3), 293-311

Wang, X. et al. 2015. Fault detection and classification for complex processes using semi-supervised learning algorithm, Chemometrics and Intelligent Laboratory Systems 149 (B), 24-32

Wang, Y. \& Zhang, Y. 2008. Multi-agent based chemical plant process monitoring and management system, Proceedings of the $4^{\text {th }}$ International Conference on Wireless Communications, Networking and Mobile Computing, 1-4

Zhong, J.H. et al. 2018. Fault diagnosis of rotating machinery based on multiple probabilistic classifiers, Mechanical System and Signal Processing 108, 99114 